\title{
La economía popular y solidaria y las finanzas populares y solidarias en Ecuador
}

\section{The popular and solidarity economy and the popular and solidarity finances in Ecuador}

\author{
Luis Armijo Auquilla Belema ${ }^{1 *}$, Álvaro Andrés Auquilla Ordóñez ${ }^{2}$ y Elsa Flor Ordóñez Bravo ${ }^{2}$ \\ ${ }^{1}$ Universidad Estatal Amazónica \\ ${ }^{2}$ Escuela Superior Politécnica de Chimborazo \\ *luisauquilla10@gmail.com
}

DOI: https://doi.org/10.26871/killkana_social.v2i3.318

\begin{abstract}
Resumen
La Economía Popular y Solidaria (EPS) y las Finanzas Populares y Solidarias (FPS) surgen en Ecuador con la administración del gobierno conocido como de la Revolución Ciudadana (15 de enero de 2007 - 24 de mayo de 2017), a tal punto que, la EPS se halla inserta en la actual Constitución de la República del Ecuador 2008. A fin de dotarle a la EPS de su marco regulatorio, la Asamblea Nacional Constituyente en 2011 aprobó la Ley Orgánica de la Economía y Popular y Solidaria y del Sector Financiero Popular y Solidario (LOEPS); en esta consta la base legal para que se hayan creado los agentes institucionales: Corporación Nacional de Finanzas Populares y Solidarias (CONAFIPS) en 2011 y Superintendencia de Economía Popular y Solidaria (SEPS) en 2012. A más del que estaba creado Instituto Nacional de Economía Popular y Solidara (IEPS) en 2009, encargado del progreso de la EPS. La CONAFIPS es la que otorga financiamiento a las organizaciones de las FPS, y éstas a la vez, financian el desarrollo de los emprendimientos de la EPS en las parroquias urbanas y rurales del país; y la SEPS, se encarga del control de las organizaciones de la EPS y de las FPS. El objetivo de estos agentes institucionales es, velar por la sostenibilidad de los emprendimientos que se desarrollan en las parroquias urbanas y rurales del país. Sin embargo, no han logrado cumplir el fin para lo que fueron creados, debido a la forma como están organizados actualmente. Los resultados así lo demuestran sobre todo en las parroquias rurales, ya que la pobreza por Necesidades Básicas Insatisfechas (NBI) según el Instituto Nacional de Estadística y Censos (INEC) a diciembre de 2017 alcanzó el 56,1\%.
\end{abstract}

Palabras clave: economía popular y solidaria, finanzas populares y solidarias, necesidades básicas insatisfechas.

\begin{abstract}
The Popular and Solidarity Economy (EPS) and the Popular and Solidarity Finances (SPF) arise in Ecuador with the administration of the government known as the Citizen Revolution (January 15, 2007 - May 24, 2017), to such an extent that, The EPS is inserted in the current Constitution of the Republic of Ecuador 2008. In order to provide the EPS with its regulatory framework, the National Constituent Assembly in 2011 approved the Organic Law of the Economy and Popular and Solidarity and the Popular Financial Sector and Solidarity (LOEPS); This is the legal basis for the creation of the institutional agents: The National Popular Finance and Solidarity Corporation (CONAFIPS) in 2011 and the Superintendence of Popular and Solidarity Economy (SEPS) in 2012. In addition to the one created by the National Institute of Economics Popular and Solidarity (IEPS) in 2009, in charge of the progress of the EPS. The CONAFIPS is the one that grants financing to the organizations of the FPS, and these at the same time, finance the development of the enterprises of the EPS in the urban and rural parishes of the country; and the SEPS, is in charge of the control of the organizations of the EPS and the FPS. The objective of these institutional agents is to ensure the sustainability of the undertakings that take place in the urban and rural parishes of the country. However, they have not managed to fulfill the purpose for which they were created, due to the way they are currently organized. The results show this especially in the rural parishes, since the poverty for Unsatisfied Basic Needs (NBI) according to the National Institute of Statistics and Censuses (INEC) to December 2017 reached $56.1 \%$
\end{abstract}

Key words: popular and solidary economy, popular and solidarity finances, unsatisfied basic needs. 


\section{Introducción}

El Ecuador es hoy en día un interesante ejercicio de diseño y aplicación de políticas públicas, cuya finalidad explícita figura en disminuir las dinámicas de la pobreza en el país. Según el Instituto Nacional de Estadística y Censos (INEC) en el año 2006 este indicador alcanzó el 37,6\%. A partir del 2007 con la llegada del gobierno conocido como de la Revolución Ciudadana, y en la lucha por descender este por ciento se han implementado diversas políticas en beneficio de los sectores populares y solidarios; la Economía Popular y Solidaria (EPS) y las Finanzas Populares y Solidarias (FPS) constituyen unas de ellas.

El alcance de la EPS y de las FPS en Ecuador, queda sustentado en la Ley Orgánica de la Economía Popular y Solidaria y del Sector Financiero Popular y Solidario (LOEPS), la cual entra en vigor en el año 2011. A fin de proporcionar sostenibilidad a la EPS y a las FPS, se han creado agentes institucionales como la Corporación Nacional de Finanzas Populares y Solidarias (CONAFIPS) en 2011, la Superintendencia de la Economía Popular y Solidaria (SEPS) en 2012, y el que estaba creado Instituto Nacional de Economía Popular y Solidaria (IEPS) en 2009, el cual funciona adscrito al Ministerio de Inclusión Económica y Social (MIES).

El IEPS es la institución encargada de velar por el desarrollo de los emprendimientos de la EPS; la CONAFIPS es el organismo que se encarga de transferir los recursos económicos a las organizaciones de las FPS (cooperativas de ahorro y crédito, entidades asociativas o solidarias, cajas y bancos comunales, y las cajas de ahorro), y estas a la vez, deben conceder crédito a las organizaciones de la EPS (sectores comunitarios, asociativos, cooperativistas y unidades económicas populares) para el financiamiento de los emprendimientos locales; y la SEPS actúa como organismo de control de la EPS y de las FPS.

Lamentablemente, estos agentes institucionales no están cumpliendo la misión para lo que fueron creados, es decir, velar por la sostenibilidad de los emprendimientos que se desarrollan al interior de la EPS, existe divorcio entré ellos. Por esta situación, se han visto truncadas las aspiraciones de potenciales emprendedores locales. Además, un factor esencial es la falta de coordinación entre la EPS y las FPS.

\section{Marco Teórico}

La economía social constituye el antecedente de la economía popular y solidaria. Según Romero (2010), la economía social data del siglo XIX en Europa y fue utilizada por primera vez por los movimientos obreros cristianos, socialistas y anarquistas.

En la última década se registra en América Latina la presencia creciente de prácticas y discursos asociados a algunos de estos nombres: economía solidaria, economía de la solidaridad, economía comunitaria, economía popular, economía social, economía social y solidaria, economía del trabajo, economía alternativa, economía plural, otra economía. Mientras que en Ecuador se le conoce como la EPS.

A fin de dotarle su marco jurídico a la EPS, la Asamblea Nacional Constituyente en 2011 aprobó la Ley Orgánica de la Economía Popular y Solidaria y del Sector Financiero Popular y Solidario (LOEPS), y esta consta la base legal para el funcionamiento de la EPS y de las FPS. A partir de la antes mencionada ley se define a la EPS.

Se entiende por Economía Popular y Solidaria a la forma de organización económica, donde sus integrantes, individual o colectivamente, organizan y desarrollan procesos de producción, intercambio, comercialización, financiamiento y consumo de bienes y servicios, para satisfacer necesidades y generar ingresos, basadas en relaciones de solidaridad, cooperación y reciprocidad, privilegiando al trabajo y al ser humano como sujeto y fin de su actividad, orientada al Buen Vivir, en armonía con la naturaleza, por sobre la apropiación, el lucro y la acumulación de capital (LOEPS, 2011, p.2-3).

Para Auquilla (2014), la economía popular y solidaria es la vía que tiene la población excluida económica y socialmente, que a través de la organización desarrollan procesos de producción, distribución, circulación y consumo, lo que les permite generan ingresos con los cuales satisfacen sus necesidades humanas en forma solidaria, con orientación al Buen Vivir. En esta definición se incluyen aquellos elementos fundamentales que definen a esta economía.

Esta forma de organización de la economía está integrada por las organizaciones conformadas en los sectores comunitarios, asociativos y cooperativistas, así como también por las unidades económicas populares (LOEPS, 2011).

En la actualidad, la crisis del sistema financiero como la expresión más directa del modelo de desarrollo capitalista, ha hecho que las diferentes formas producción, distribución, circulación y consumo de los sectores populares y solidarios que no funcionan con la lógica de acumulación del capital sino de la organización, cobren fuerza e importancia en la construcción de otro modelo de desarrollo económico y social, el de la solidaridad.

En trabajos de Orellana (2009), Cardoso, Bermeo y Fresa (2012) y Vaca (2012) se hallan aportes sobre las FPS. Siendo el de mayor relevancia el tributado por Cardoso, Bermeo y Fresa (2012), quienes manifiestan que:

Las finanzas populares y solidarias es el conjunto de ideas, esfuerzos, capacidades, apoyos, normas, programas, instrumentos, recursos y estructuras, que actúan en cada situación geográfica definida y limitada (recinto, parroquia, barrio suburbano y otros) con el propósito de que la población organice el mercado del ahorro, del crédito y de los servicios financieros en su propio beneficio y en pos del desarrollo de toda la comunidad, abiertos al intercambio de productos y servicios con otras localidades, en perspectiva de construir un nuevo sistema de flujos que tengan al ser 
humano como centro del desarrollo económico y social (p. 7).

En opinión de Auquilla (2014), las finanzas populares y solidarias constituyen la nueva estructura financiera que está al servicio de los sectores de bajos recursos económicos, quienes han sido excluidos del financiamiento por la banca tradicional y que actúan en forma solidaria.

Además, a las FPS se las considera como las finanzas del pueblo, ya que en la actualidad la mayor parte de la población cultural y éticamente rica y sana sufre por la pobreza económica. Sin embargo, los pobres crean riqueza para otros con su trabajo, producen bienes y servicios y al mismo tiempo generan ahorros. Muchas personas de los sectores populares y solidarios especialmente rurales, comienzan a entender que ellos mismos deben administrar los excedentes que generan.

Las FPS se hallan integradas por las cooperativas de ahorro y crédito, las entidades asociativas o solidarias, las cajas y bancos comunales, y las cajas de ahorro (LOEPS, 2011).

La Superintendencia de Economía Popular y Solidaria (SEPS), es una entidad técnica de supervisión y control de las organizaciones EPS y de las FPS, con personalidad jurídica de derecho público y autonomía administrativa y financiera, que busca el desarrollo, estabilidad, solidez y correcto funcionamiento del sector económico popular y solidario (2014).

A partir de 2012, todas las nuevas organizaciones de la EPS, así como de las FPS, deben inscribirse en la SEPS para que inicien con su vida jurídica, y más aun las que ya estén constituidas (Reglamento LOEPS, 2012).

Las organizaciones de las parroquias urbanas y rurales para financiar los emprendimientos que se desarrollan al interior de la EPS, deben solicitar financiamiento a las organizaciones de las FPS, lamentablemente estas no cuentan con suficientes recursos para conceder créditos y financiar los entendimientos locales, excepto las cooperativas de ahorro y crédito; mientras que, las entidades asociativas o solidarias, las cajas y bancos comunales, y las cajas de ahorro deben acudir a la Corporación Nacional de Finanzas Populares y Solidarias (CONAFIPS) a solicitar financiamiento, la cual opera como intermediario.

\section{Metodología}

Una vez examinados los elementos teóricos referentes al tema abordado, los autores consideraron de suma importancia la puesta en práctica de los mismos en un caso de estudio. La investigación se realizó en las organizaciones del Gobierno Autónomo Descentralizado (GAD) de la parroquia rural San José. Este gobierno parroquial se halla integrado políticamente por nueve localidades: Cajabamba 1, Cajabamba 2, Ceslao Marín, El Carmen, La Esperanza, San José (cabecera parroquial), San José 2, San Vicente y Tsumashunchi (ver figura 1).

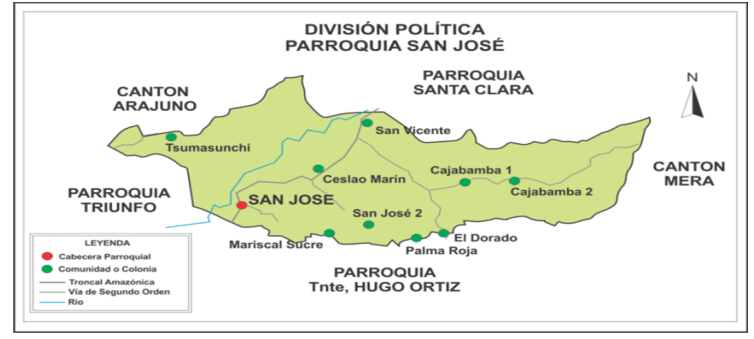

Figura 1. Mapa político de la parroquia rural San José

Fuente: GAD de la parroquia rural San José

El clima es ecuatorial, posee una temperatura entre 18 y 24 grados durante todo el año, con una precipitación promedio anual que supera los $3.000 \mathrm{~mm}$, la humedad oscila entre 87 y $89 \%$, el bosque es húmedo pluvial pre montano, la topografía es irregular, el suelo está formado por sedimentos de arcilla y areniscas ligeramente gredoso y de poco drenaje, poco profundos (GAD de la parroquia rural San José , 2015).

EL GAD de la parroquia rural San José se encuentra ubicado en la región amazónica de Ecuador, al noroccidente de la provincia de Pastaza (la más extensa en territorio con $29.520 \mathrm{~km}^{2}$, de estos le corresponde $69 \mathrm{~km}^{2}$ al GAD San José), a $21 \mathrm{~km}$ del cantón Pastaza, y a 12 km del cantón Santa Clara (ver figura 2).

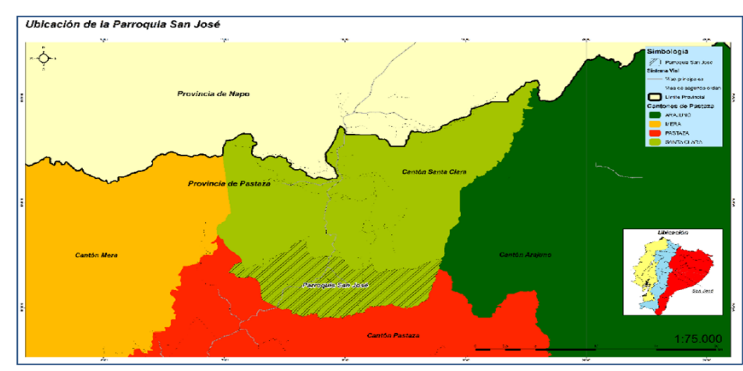

Figura 2. Ubicación de la parroquia rural San José Fuente: GAD de la parroquia rural San José

Según el Instituto Nacional de Estadística y Censos (2010), el GAD San José tiene 735 habitantes, de esta población, 386 son hombres y 349 mujeres. Se destaca que el 49,66\% de la población, entre 18 y 65 años se encuentran en capacidad de trabajar.

El número de emprendimientos que se ejecutan en las localidades del GAD de la parroquia rural San José son 25 y se hallan distribuidos como siguen (Ver tabla 1). 
Tabla 1 Emprendimientos en el GAD San José

\begin{tabular}{|l|c|c|l|l|l|}
\hline Localidades & $\mathbf{N}^{\circ}$ & \multicolumn{5}{|c|}{ Emprendimientos } \\
\hline Cajabamba 1 & 2 & 2 & Agrícolas & & \\
\hline Cajabamba 2 & 3 & 3 & Agrícolas & & \\
\hline Ceslao Marín & 3 & 3 & Agrícolas & & \\
\hline San José (cabecera parroquial) & 6 & 4 & Agrícolas & 2 & Pecuarios \\
\hline San Vicente & 5 & 3 & Agrícolas & 2 & Pecuarios \\
\hline Tsumashunchi & 2 & 2 & Agrícolas & & \\
\hline El Carmen & 2 & 2 & Agrícolas & & \\
\hline La Esperanza & 1 & 1 & Agrícola & & \\
\hline San José 2 & 1 & 1 & Agrícola & & \\
\hline Total & 25 & 21 & Agrícolas & 4 & Pecuarios \\
\hline
\end{tabular}

Fuente: Elaboración propia a partir de la encuesta de abril-mayo de 2012

A partir de las principales características de las localidades del GAD San José, se evidencia la ejecución de emprendimientos agrícolas y pecuarios, se destaca San José como cabecera parroquial y San Vicente como las localidades en las que más se desarrollan los emprendimientos agropecuarios.

Para diagnosticar el problema de la investigación, se realizaron talleres participativos con 75 dirigentes que se hallan involucrados en los 25 emprendimientos que se desarrollan en las localidades del GAD de la parroquia rural San José: presidente, secretario y tesorero, siendo estos actores directos en la investigación. El criterio de selección de los dirigentes se basó, en que estos son los responsables directos de la administración de dichos emprendimientos.

Por lo que, la muestra se seleccionó de manera intencional a los 75 dirigentes, por tanto, técnicamente se considera una muestra no probabilística. Por tratarse de un estudio cualitativo, el tamaño de la muestra no es importante desde una perspectiva probabilística, pues, el interés no es generalizar los resultados a una población más amplia, ya que lo que se busca es profundidad; motivo por el cual, se pretende calidad más que cantidad, en donde, lo fundamental es la aportación de personas, participantes, organizaciones, eventos, hechos y demás, que ayuden a entender el fenómeno de estudio, y a responder a las preguntas de investigación que se han planteado (Hernández, Fernández, y Baptista, 2010).

Como resultado de estos talleres se logró elaborar una encuesta. A fin de validar y elaborar la definitiva para su ulterior aplicación, se realizó una prueba piloto con 25 dirigentes, para finalmente aplicar la encuesta a los 75 directivos.

Para el análisis de fiabilidad de la encuesta se utilizó el coeficiente Alfa de Cronbach. El procesamiento fue realizado con el paquete estadístico SPSS, el cual arrojó un valor de 0,955 ; lo que evidencia una alta confiabilidad de la encuesta aplicada.

\section{Resultados}

En el desempeño actual de la EPS, las FPS ocupan un sitio fundamental por constituir la base financiera para la realización de las actividades productivas que se desarrollan mediante los emprendimientos. Sin embargo, el funcionamiento actual de la EPS y las FPS no están cumpliendo sus objetivos para el fortalecimiento de los emprendimientos. En consecuencia, se considera que se están apartando los potenciales emprendedores debido a la falta de coordinación directa, y a una adecuada organización entre las entidades creadas al respecto. En la figura 3, se ilustra cómo están estructuradas actualmente la EPS y las FPS.

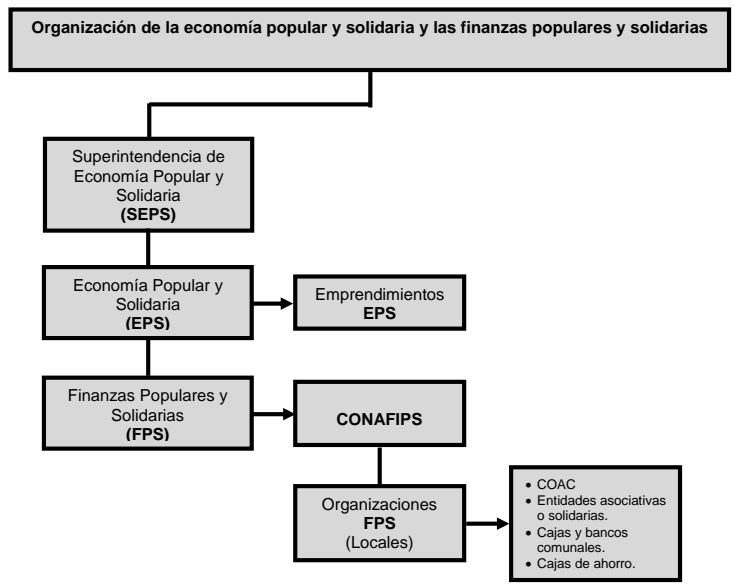

Figura 3. Funcionamiento actual entre EPS y las FPS Fuente: Auquilla, L. (2014)

Esta forma de organización ha implicado una cadena de trámites muy larga para poder acceder al financiamiento por parte de los emprendimientos generados. Como resultado, un emprendimiento que se estable por primera vez y que su aspiración es ser miembro de la EPS, debe inscribirse y recibir la carta de aceptación de la SEPS para iniciar con su vida jurídica; una vez que ha recibido la aprobación de esta entidad, pasa a ser organización de la EPS.

El siguiente paso consiste en acudir al Instituto de Economía Popular y Solidaria (IEPS) provincial -en este caso de Pastaza- institución que está a cargo de la EPS, para solicitar asesoría sobre el posible emprendimiento a ejecutar en la localidad a la cual pertenece la organización recién constituida, y si posee el proyecto, debe presentarlo a las autoridades del IEPS provincial a fin de que sea analizado, y si procede reciba la aprobación. En ambos casos, el IEPS debe ofrecer los servicios de capacitación, seguimiento y evaluación al posible emprendimiento local a desarrollar. 
Si el emprendimiento presentado por la organización ha recibido la aceptación del IEPS provincial, se solicitan los servicios financieros de las organizaciones de las FPS como eslabón básico para su desarrollo. Son las organizaciones FPS de las localidades las que brindan el crédito para el financiamiento de los emprendimientos locales; pero, éstas (las entidades asociativas o solidarias, las cajas y bancos comunales, y las cajas de ahorro) no conceden el financiamiento si no reciben los recursos de la CONAFIPS, no ocurre lo mismo con las cooperativas de ahorro y crédito, ya que cuentan con recursos propios y suficientes para conceder créditos a los emprendedores de las parroquias urbanas y rurales, sobre todo de las primeras.

A partir de este funcionamiento entre la EPS y de las FPS se derivan una serie de limitantes que frenan el progreso de los emprendimientos en las localidades del GAD de la parroquia rural San José, y por tanto, actúan en detrimento del aumento de la calidad de vida de sus habitantes.

A la EPS y las FPS les corresponde estimular nuevas iniciativas de emprendimiento, dar capacitación con contenidos y objetivos más ligados a las necesidades de cada momento y cada lugar, lo que no está ocurriendo debido a la organización actual. Se debe incentivar a los emprendedores con la entrega de servicios financieros ágiles, así como dotarles de asesoramiento y capacitación para el desarrollo de los emprendimientos locales, pero los mismos no son proporcionados.
A continuación, se presenta los resultados más relevantes de las encuestas realizadas:

En las localidades del GAD de la parroquia rural San José predomina la población con nivel de instrucción primaria con el $72 \%$, por lo que se considera que a los habitantes se les debe dar asesoramiento, capacitación y acompañamiento a los proyectos, a fin de que los emprendimientos de la EPS sean sostenibles financiera y socialmente (ver tabla 2).

Los encuestados en un $84 \%$ han confirmado que el agente institucional IEPS provincial, no ha cumplido con el cometido de dar asesoramiento a las organizaciones locales para el fomento de los emprendimientos de la EPS (ver tabla 3).

La capacitación tampoco ha llegado a las organizaciones de la EPS así lo confirman el 85,3\% de los indagados, por lo que, el desarrollo de los emprendimientos locales se ha visto limitados por falta de formación (ver tabla 3).

Los pesquisados han declarado en un $76 \%$ que no se encuentra satisfechos con las condiciones de financiamiento que le ofrecen las organizaciones de las finanzas populares y solidarias para la ejecución de los emprendimientos (ver tabla 3)

También han manifestado que no han hecho uso de las organizaciones de las FPS en un $82,7 \%$, por considerar el manejo de mucha burocracia al momento de otorgar el financiamiento, así como, la falta de comunicación y transparencia de la información al respecto (ver tabla 4).

Resultados cuantitativos de la encuesta aplicada:

Tabla 2 Características de los encuestados

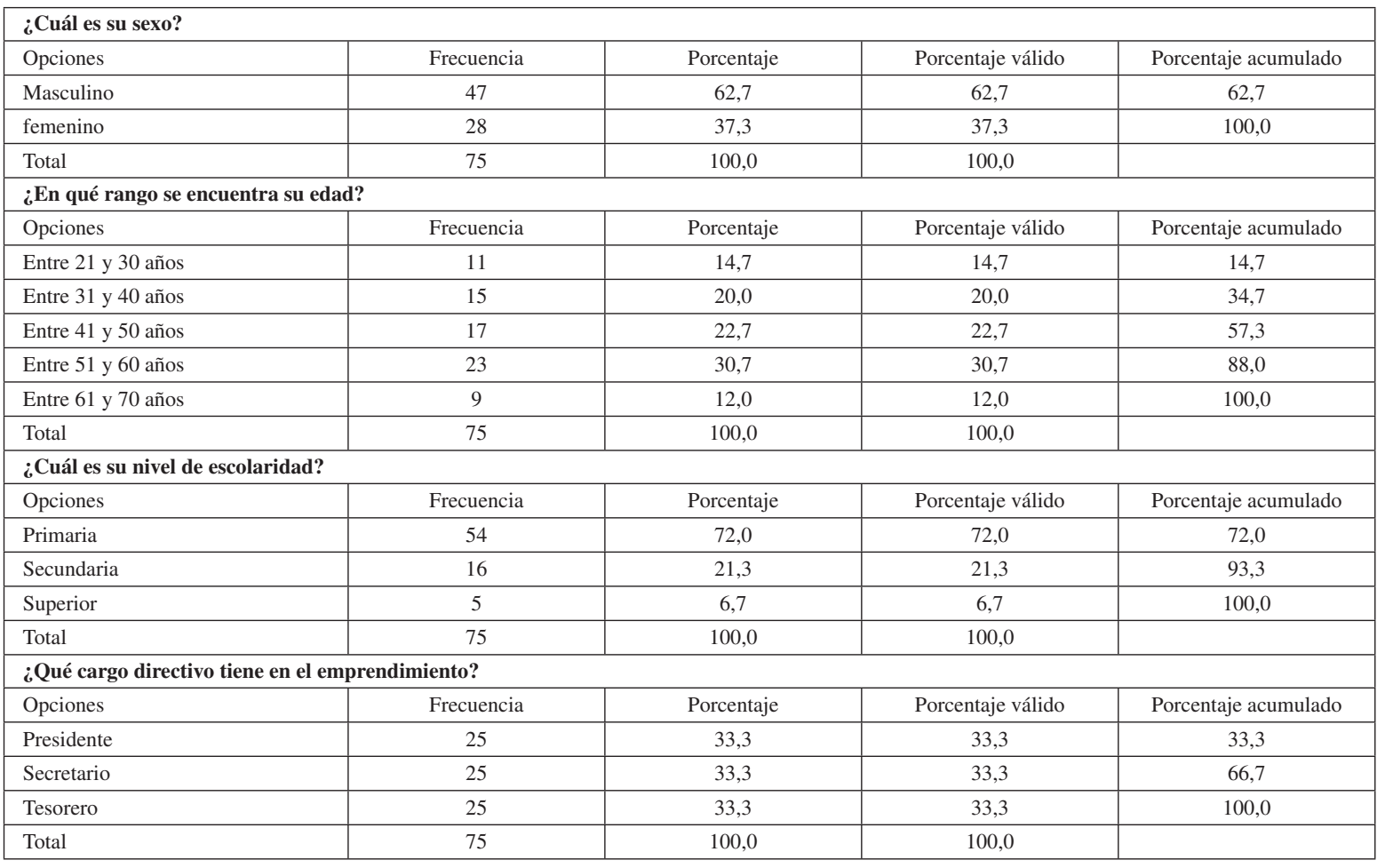

Fuente: Elaboración propia a partir de la encuesta de abril-mayo de 2012 
Tabla 3 Agentes institucionales

\begin{tabular}{|c|c|c|c|c|c|c|}
\hline \multirow{2}{*}{ Preguntas } & \multicolumn{2}{|c|}{ Insatisfecho } & \multicolumn{2}{|c|}{ Medianamente satisfecho } & \multicolumn{2}{|c|}{ Completamente satisfecho } \\
\hline & Frecuencia & $\%$ & Frecuencia & $\%$ & Frecuencia & $\%$ \\
\hline $\begin{array}{l}\text { ¿Cómo dirigente de la organización, usted se encuentra satisfecho } \\
\text { con el asesoramiento que le brinda el instituto de economía popular } \\
\text { y solidaria de la provincia de Pastaza para el fomento de los } \\
\text { emprendimientos? }\end{array}$ & 63 & 84 & 9 & 12 & 3 & 4 \\
\hline $\begin{array}{l}\text { ¿Cómo dirigente de la organización, usted se encuentra satisfecho } \\
\text { con la capacitación que le brinda el instituto de economía popular } \\
\text { y solidaria de la provincia de Pastaza para el desarrollo de los } \\
\text { emprendimientos? }\end{array}$ & 64 & 85,3 & 8 & 10,7 & 3 & 4 \\
\hline $\begin{array}{l}\text { ¿Cómo dirigente de la organización, usted se encuentra satisfecho } \\
\text { con las condiciones de financiamiento que le ofrecen las finanzas } \\
\text { populares y solidarias para la ejecución de los emprendimientos? }\end{array}$ & 57 & 76 & 13 & 17,3 & 5 & 6,7 \\
\hline
\end{tabular}

Fuente: Elaboración propia a partir de la encuesta de abril-mayo de 2012

Tabla 4 Finanzas populares y solidarias

\begin{tabular}{|c|c|c|c|c|c|c|}
\hline \multirow{2}{*}{ Preguntas } & \multicolumn{2}{|c|}{ Insatisfecho } & \multicolumn{2}{|c|}{ Medianamente satisfecho } & \multicolumn{2}{|c|}{ Completamente satisfecho } \\
\hline & Frecuencia & $\%$ & Frecuencia & $\%$ & Frecuencia & $\%$ \\
\hline $\begin{array}{l}\text { ¿La organización de la cual usted es dirigente, ha hecho uso de las } \\
\text { finanzas populares y solidarias para el inicio de los emprendimien- } \\
\text { tos que se ejecutan en su localidad? }\end{array}$ & 62 & 82,7 & 6 & 8 & 7 & 9,3 \\
\hline $\begin{array}{l}\text { ¿Cómo directivo de la organización, se implica y participa activa- } \\
\text { mente en las finanzas populares y solidarias de la organización? }\end{array}$ & 62 & 82,7 & 6 & 8 & 7 & 9,3 \\
\hline $\begin{array}{l}\text { ¿La información sobre las finanzas populares y solidarias de la } \\
\text { organización es pública y accesible para los diferentes grupos de } \\
\text { interés relacionados con la organización? }\end{array}$ & 70 & 93,3 & 2 & 2,7 & 3 & 4 \\
\hline $\begin{array}{l}\text { ¿La organización de la economía popular y solidaria de la cual } \\
\text { usted es directivo, realiza un diagnóstico antes de iniciar con la } \\
\text { ejecución de un emprendimiento en su localidad? }\end{array}$ & 72 & 96 & 1 & 1,3 & 2 & 2,7 \\
\hline $\begin{array}{l}\text { ¿La organización de la economía popular y solidaria de la cual } \\
\text { usted el dirigente, analiza líneas de crédito para favorecer la } \\
\text { sostenibilidad económica de sus emprendimientos? }\end{array}$ & 69 & 92 & 3 & 4 & 3 & 4 \\
\hline $\begin{array}{l}\text { ¿La organización de la economía popular y solidaria de la cual } \\
\text { usted es directivo, aplica estrategias de desarrollo local? }\end{array}$ & 71 & 94,7 & 1 & 1,3 & 3 & 4 \\
\hline $\begin{array}{l}\text { ¿La organización de la economía popular y solidaria de la cual } \\
\text { usted es dirigente, emplea la cadena de valor? }\end{array}$ & 68 & 90,7 & 4 & 5,3 & 3 & 4 \\
\hline $\begin{array}{l}\text { ¿La organización de la economía popular y solidaria de la cual } \\
\text { usted es directivo, utiliza mecanismos de control de beneficiarios? }\end{array}$ & 69 & 92 & 2 & 2,7 & 4 & 5,3 \\
\hline $\begin{array}{l}\text { ¿La organización de la economía popular y solidaria de la cual } \\
\text { usted es dirigente, administra modalidades de desembolso? }\end{array}$ & 69 & 92 & 3 & 4 & 3 & 4 \\
\hline $\begin{array}{l}\text { ¿La organización de la economía popular y solidaria de la cual } \\
\text { usted es directivo, emplea planificación del financiamiento? }\end{array}$ & 68 & 90,7 & 4 & 5,3 & 3 & 4 \\
\hline $\begin{array}{l}\text { ¿El instituto de economía popular y solidaria de la provincia de } \\
\text { Pastaza, da seguimiento a los emprendimientos que se ejecutan en } \\
\text { su localidad? }\end{array}$ & 57 & 76 & 13 & 17,3 & 5 & 6,7 \\
\hline $\begin{array}{l}\text { ¿Las organizaciones de las finanzas populares y solidarias de la } \\
\text { provincia de Pastaza, realizan evaluaciones de los recursos finan- } \\
\text { cieros entregados a la organización de la cual usted es dirigente? }\end{array}$ & 65 & 86,7 & 7 & 9,3 & 3 & 4 \\
\hline
\end{tabular}

Fuente: Elaboración propia a partir de la encuesta de abril-mayo de 2012

A partir de los resultados de la encuesta aplicada se evidencia que, de la forma como están organizados la EPS y las FPS no están proporcionando ninguna ayuda a los emprendedores locales. Es necesario que la EPS y las FPS cumplan el objetivo de su creación que consta en la LOEPS; es decir, una vez que se hayan identificado a futuros emprendedores se les otorgue facilidades para que puedan ejecutar los emprendimientos o continúen desarrollando los iniciados, y puedan constituirse en los artífices para disminuir la pobreza a nivel nacional y la pobreza por Necesidades Básicas Insatisfechas (NBI) que se posee actualmente el país.
Según el INEC (2017), el índice de pobreza a nivel nacional alcanzó el 21,5\%. La pobreza por NBI nivel nacional llegó a $31,8 \%$, a nivel urbano a $20,5 \%$ y a nivel rural a $56,1 \%$. Por lo que, las parroquias rurales siguen siendo las más afectadas.

La pobreza por NBI es una medida de pobreza multidimensional desarrollada en los años 80 por la Comisión Económica para América Latina y el Caribe (CEPAL). El método abarca cinco dimensiones y dentro de cada dimensión existen indicadores que miden privaciones como: 
a. Capacidad económica. El hogar se considera privado de esta dimensión si: i) los años de escolaridad del jefe (a) de hogar es menor o igual a 2 años y, ii) existen más de tres personas por cada persona ocupada del hogar.

b. Acceso a educación básica. El hogar se considera privado en esta dimensión si: existen en el hogar niños de 6 a 12 años de edad que no asisten a clases.

c. Acceso a vivienda. El hogar está privado si: i) el material del piso es de tierra u otros materiales o, ii) el material de las paredes son de caña, estera u otros.

d. Acceso a servicios básicos. La dimensión considera las condiciones sanitarias de la vivienda. El hogar es pobre si: i) la vivienda no tiene servicio higiénico o si lo tiene es por pozo ciego o letrina o, ii) si el agua que obtiene la vivienda no es por red pública o por otra fuente de tubería.

e. Hacinamiento. El hogar se considera pobre si la relación de personas por dormitorio es mayor a tres.

\section{Conclusiones}

Los fundamentos teóricos para el estudio de la economía popular y solidaria, se han venido conformando a partir de los análisis sobre la economía social en Europa surgida desde el siglo XIX; así como también, de los aportes conceptuales y prácticos de esta economía que se registran en la última década en América Latina.

El fomento del sector de la economía popular y solidaria constituye un punto de partida para experimentar desde abajo el proceso de trasformación del trabajador asalariado en productor asociado.

La crisis del sistema financiero como la expresión más directa del modelo de desarrollo capitalista en la actualidad, ha hecho que las finanzas populares y solidarias cobren fuerza e importancia, en donde se debe dejar de hablar de microfinanzas para asumir la categoría de finanzas populares y solidarias.

La organización actual en la que se halla la economía popular y solidaria y las finanzas populares y solidarias, no está aportando mayormente para el desarrollo de los emprendimientos locales.

\section{Recomendaciones}

Se debe conceder recursos financieros en forma directa a las entidades asociativas o solidarias, a las cajas y bancos comunales, y las cajas de ahorro, sin que participe como intermediario la Corporación Nacional de Finanzas Populares y Solidarias (CONAFIPS).

Se hace necesario diseñar una propuesta de coordinación entre la economía popular y solidaria (EPS) y las finanzas populares y solidarias (FPS), de manera que contribuya a garantizar las condiciones para la sostenibilidad financiera y social de los emprendimientos locales.

\section{Referencias Bibliográficas}

Auquilla, L.
$y$ solidaria $y$ la coordinación con las

finanzas populares y solidarias en ecuador Descargado de http://docplayer.es/ 26790873-Universidad-de-la-habana -facultad-de-economia-departamento -de-ciencias-empresariales.html\# show_full_text

Cardoso, G., Bermeo, E., y Fresa, M. (2012). Ecuador: Economía y finanzas populares y solidarias, para el buen vivir. Quito: Editorial Imprefepp.

GAD de la parroquia rural San José . (2015). Actualización del plan de desarrollo y ordenamiento territorial fase 1 diagnóstico.

Hernández, R., Fernández, C., y Baptista, M. (2010). Metodología de la investigación. México D. F.: Editorial McGraw-Hill.

Instituto Nacional de estadística y Censos . (2010). Censo de población y vivienda. Descargado de http://redatam.inec.gob.ec/ cgibin/RpWebEngine. exe/PortalAction ? \&MODE $=$ MAIN\&BASE $=\mathrm{CPV} 2010 \& \mathrm{MAIN}=$ WebServerMain.inl

Instituto Nacional de estadística y Censos . (2017). Encuesta nacional de empleo, desempleo y subempleo. indicadores de pobreza y desigualdad. Descargado de http: / / www.ecuadorencifras.gob.ec/ documentos/webinec/POBREZA/2017/ Diciembre/122017_Pobreza20pdf.pdf

LOEPS. (2011). Quito: Editora Nacional. (Registro Oficial No. 444)

Orellana, E. (2009). Las finanzas sociales y solidarias en el ecuador: Verdades y desafios. Quito: Editorial Universidad Politécnica Salesiana de Ecuador.

Reglamento LOEPS. (2012). Quito: Editora Nacional. (Registro Oficial No. 648)

Vaca, J. (2012). Reflexiones conceptuales y propuestas sobre micro-finanzas y su relación con finanzas populares y solidarias. Quito: Editorial Red Financiara Rural.
Recibido: 30 de junio de 2018

Aceptado: 3 de septiembre de 2018 
